\title{
Chloroquine Decreases Cardiomyocyte Autophagy and Improves Cardiac Function in a Mouse Model of Duchenne Muscular Dystrophy
}

\author{
Takaya Hirata \\ Kyoto University \\ Kentaro Akagi \\ Kyoto University \\ Daisuke Yoshinaga \\ Kyoto University \\ Katsutsugu Umeda \\ Kyoto University \\ Souichi Adachi \\ Kyoto University \\ Toshio Heike \\ Kyoto University \\ Junko Takita \\ Kyoto University
}

Shiro Baba ( $\nabla$ shibaba@kuhp.kyoto-u.ac.jp)

Kyoto University https://orcid.org/0000-0001-6253-6087

\section{Research}

Keywords: Duchenne muscular dystrophy, autophagy, mdx mice, Chloroquine

Posted Date: January 19th, 2021

DOI: https://doi.org/10.21203/rs.3.rs-149383/v1

License: (c) (1) This work is licensed under a Creative Commons Attribution 4.0 International License. Read Full License 


\section{Abstract}

Background: Duchenne muscular dystrophy (DMD), a severe degenerative skeletal and cardiac muscle disease, has a poor prognosis, and no curative treatments are available. Because autophagy has been reported to contribute to skeletal muscle degeneration, therapies targeting autophagy are expected to improve skeletal muscle hypofunction. However, the role of this regulatory mechanism has not been evaluated clearly in DMD cardiomyocytes.

Methods: In the present study, we demonstrated that autophagy was enhanced in the cardiomyocytes of $m d x$ mice, a model of DMD, and that increased autophagy contributed to the development of cardiomyopathy in this context.

Results: As assessed by GFP-mRFP-LC3 transfection, autophagosomes were more abundant in cardiomyocytes of $m d x$ mice compared with control wild-type (WT) mice. The number of autophagosomes was significantly enhanced by isoproterenol-induced cardiac stress (4 weeks) in cardiomyocytes of $m d x$ but not WT mice. Simultaneously, isoproterenol increased cardiomyocyte fibrosis in $m d x$ but not WT mice. Administration of chloroquine, an autophagy inhibitor, significantly decreased cardiomyocyte autophagy and fibrosis in $m d x$ mice, even after isoproterenol treatment. Left ventricle size and function were evaluated by echocardiography. Left ventricular contraction was decreased in $m d x$ mice after isoproterenol treatment compared with control mice, which was alleviated by chloroquine administration.

Conclusions: These findings suggested that heart failure of DMD could be associated with autophagy. Therefore, autophagy inhibitors, such as chloroquine, are a potential therapeutic modality for heart failure in DMD patients.

\section{Background}

Duchenne muscular dystrophy (DMD) is the most common and severe form of muscular dystrophy, and is caused by mutations in the gene encoding dystrophin located on chromosome Xp21 [1, 2, 3]. DMD is inherited in an autosomal recessive manner and is relatively common, with an incidence of approximately 1 per 3,500 male births $[4,5]$.

Muscle degeneration and subsequent fibrosis occur at early ages in DMD patients. Muscle weakness results in walking difficulties, and ultimately in the development of respiratory muscle failure and heart failure. Respiratory and heart failure are common lethal complications of DMD, and frequently affect patients in their late teens and early twenties [1]. Recently, ventilator support devices, such as home ventilators, have been developed, prolonging the mean lifespan of ventilated DMD patients to over 35 years $[6,7,8,9,10,11]$. Therefore, cardiomyopathy is now the leading cause of death in DMD patients. The percentage of DMD patients that died from cardiac complications increased from $8-44 \%$ after the development of home ventilation devices in the 1990s [12]. 
Fibrosis-associated cardiomyopathy generally leads to dilated cardiomyopathy. More than $80 \%$ of DMD patients older than 18 years have reduced cardiac function, and $90 \%$ of DMD patients develop dilated cardiomyopathy $[13,14]$. General therapeutic protocols for dilated cardiomyopathy secondary to DMD are not curative, e.g. combinations of diuretics, vasodilators, and beta-blockers $[15,16]$. To develop more targeted approaches to treatment, it is crucial to delineate the regulatory mechanisms of cardiomyopathy in DMD. A recent report suggested that cardiomyocyte apoptosis contributes to DMD-induced cardiomyopathy in an in vitro study using induced pluripotent stem cells (iPSCs) [17]. In the present study, we focused on another type of cell death, autophagy, to determine if this regulatory mechanism contributed to DMD-induced cardiomyopathy using the $m d x$ mouse model of DMD.

\section{Materials And Methods Experimental animals}

C57BL/ 6 wild-type (WT) mice were purchased from CLEA Japan, Inc. (Japan). Mdx mice, a model of DMD, were bred at Kyoto University. All animal procedures were conducted in accordance with the guidelines of the Kyoto University Animal Committee and with prior approval from the Institutional Ethical Committee and an ARRIVE guideline. Animals were handled in accordance with the Declaration of Helsinki.

To increase cardiac load, capsulated isoproterenol was inserted subcutaneously from 12 to 16 weeks in both WT and $m d x$ mice for a dosage of $0.5 \mathrm{mg} / \mathrm{kg} /$ day. For chloroquine treatment, capsulated chloroquine was subcutaneously inserted simultaneously with isoproterenol for a dosage of $0.4 \mathrm{mg} / \mathrm{kg} /$ day. Clinical observations to monitor the side effects of all experiments were recorded every other day. Echocardiography was performed at 16 weeks of age, as was histological evaluation. A summarized animal protocol is shown in Fig. 1.

\section{Electron microscopy}

For transmission electron microscopy analysis, left ventricular sections from 16 week non-treated WT and $m d x$ mice were dissected after sacrifice and fixed for $1 \mathrm{~h}$ in a pH 7.4 solution containing $4 \%$ paraformaldehyde (PFA) and $0.5 \%$ glutaraldehyde in $0.1 \mathrm{M}$ cacodylate buffer. Ultra-thin sections were cut at a thickness of $7 \mu \mathrm{m}$ and embedded on glass slides for observation with an $\mathrm{H}-7650$ electron microscope (Hitachi, Ltd., Japan).

\section{Histology and immunohistochemistry}

Mouse hearts were isolated, and 4\%-PFA fixed left ventricles were sliced at $7 \mu \mathrm{m}$ and stained using a Hematoxylin-Eosin Stain Kit and Picro-Sirius Red Stain Kit (COSMO BIO, Japan). Fibrosis was imaged using a BZ-9000 microscope, and fibrotic areas were quantified using the automated calculation software of the BZ-9000 (KEYENCE, Japan). Transduction of green/red fluorescent protein-LC3 (GFP-mRFP-LC3) 
(COSMO BIO) was performed by lipofection into left ventricular sections. The number of green fluorescent dots, representing autophagosomes, were counted with the BZ-9000 microscope.

\section{Echocardiography}

Hemodynamics were indirectly measured by an echocardiogram with a $50 \mathrm{MHz}$ transducer (Vevo2100; Primetech, UK). Left ventricular end-diastolic and end-systolic diameter (LVDd, LVDs), end-diastolic interventricular septal thickness (IVSd), left ventricular end-diastolic posterior wall thickness (LVPWd), left ventricular fractional shortening (LVFS), and LV ejection fraction (LVEF) were measured to evaluate cardiac function in 16-week-old mice. During echocardiography, mice were sedated with 1-3\% sevoflurane (Maruishi Seiyaku, Japan), with an approximate heart rate of 400 beats per minute.

\section{Statistics}

All experiments were performed at least three times, and statistical significance was evaluated with a one-way ANOVA followed by the Tukey-Kramer test using JMP® Pro156 (11.0.0) software. $p$-values < 0.05 were considered statistically significant.

\section{Results}

\section{Increased autophagy in $\mathrm{mdx}$ mouse cardiomyocytes}

To determine if autophagy occurred in DMD cardiomyocytes, we first observed autophagosomes in cardiomyocytes of non-treated 16-week-old WT mice and $m d x$ mice using an electron microscope. (Fig. 2A) Abundant GFP-mRFP-LC3 positive dots, representing autophagosomes, were present in $m d x$ mouse cardiomyocytes. By contrast, sparse GFP-mRFP-LC3 positive dots were detected in WT mouse cardiomyocytes. (Fig. 2B (a and c)) After enhancing cardiac stress by administration of isoproterenol for 1 month, the number of GFP-mRFP-LC3 positive dots increased in cardiomyocytes of 16 week WT and $m d x$ mice. (Fig. 2B (b and d)) However, the upregulation ratio of GFP-mRFP-LC3 positive dots was dramatically enhanced by isoproterenol in cardiomyocytes of $m d x$ mice compared with those of WT mice. Subsequently, chloroquine, an autophagy inhibitor, was administered to $m d x$ mice simultaneously with isoproterenol for 1 month to verify that the GFP-mRFP-LC3 positive dots were autophagosomes. Administration of chloroquine diminished nearly all GFP-mRFP-LC3 positive dots in 16 week $m d x$ mouse cardiomyocytes. (Fig. 2B (e)) As previous papers mentioned that autophagy was over activated in $m d x$ mouse, we performed western blotting assessing LC1 and LC3. LC1 and LC3 were not clearly detected in heart sections of both $\mathrm{BL} / 7$ control mice and $\mathrm{mdx}$ mice without cardiac stress by isoproterenol [18]. (Supplementary Fig. 1) These findings indicated that autophagy occurred constitutively in $m d x$ mouse cardiomyocytes, and that $m d x$ cardiomyocytes were more sensitive to cardiac stress than WT cardiomyocytes.

Isoproterenol-induced cardiac stress increased cardiomyocyte fibrosis significantly in only mdx mice and was inhibited by chloroquine 
To further evaluate the downstream effects of cell death, the fibrotic area in the left ventricle (LV) was measured after 1 month of isoproterenol-induced cardiac stress. The LV fibrotic area in 16 week $m d x$ mice was remarkably larger than that of WT mice. (Fig. 3A ( $a$ and b)) Interestingly, simultaneous administration of chloroquine with isoproterenol significantly inhibited LV fibrosis in 16 week $m d x$ mice. (Fig. 3A (c)) The calculated fibrosis area was also significantly larger in the LV of $m d x$ mice. No mice treated with chloroquine exhibited side effects during the study. These findings indicated that cardiac stress significantly induced cardiac regeneration in $m d x$ mice by accelerated autophagy.

\section{Chloroquine alleviated impaired cardiac contraction in mdx mice}

To explore the role of autophagy in $m d x$ mouse cardiac function, we measured the key echocardiographic markers ILVDd, LVDs, LVFS, LVEF, IVSd, and LVPWd after treatment or non-treatment with isoproterenol with or without chloroquine, as shown in Fig. 1. LVDd, LVDs, IVSd, and LVPWd were not significantly affected by isoproterenol or chloroquine in WT or $m d x$ mice. (Fig. 4A, B, E, F) However, LVFS and LVEF, markers of cardiac contraction, were significantly decreased by isoproterenol treatment in $m d x$ mice. Reductions of LVFS and LVEF in $m d x$ mice were alleviated by simultaneous administration of chloroquine with isoproterenol. (Fig. 4C, D) Major well-characterized side effects of chloroquine, including vomiting and weight-loss, were not observed in WT or $m d x$ mice. These results indicated that inhibiting autophagy with chloroquine protected cardiac function in isoproterenol-stressed $m d x$ mice.

\section{Discussion}

In the present study, we demonstrated that autophagy is a major contributing mechanism to cardiomyopathy in the $m d x$ mouse model of DMD, and is accompanied by reduced cardiac contraction.

Although cardiomyopathy is a leading cause of death in DMD patients, effective therapies to prevent or slow the progression of disease are not available $[15,16]$. Because the regulatory mechanisms of cardiomyopathy remain incompletely understood, current approaches to treatment of cardiomyopathy are palliative therapies, including diuretics, angiotensin-converting enzyme inhibitors, and beta-blockers.

Recent reports have demonstrated that the mean lifespan of DMD patients is as high as 35 years of age, and that more than half of DMD patients die from cardiomyopathy $[12,13,14]$. To improve the prognosis of DMD-related cardiomyopathy, mechanistic studies to yield more targeted approaches to treatment are necessary. In the present study, we focused on autophagic cardiomyocyte cell death using the $m d x$ mouse model of DMD.

Previous reports suggested that skeletal muscle autophagy is decreased in DMD patients and $m d x$ mice. As a result of this mechanism, waste products accumulate in the cytosol, impairing skeletal muscle function $[19,20,21]$. Unexpectedly, in the present study, we identified that cardiomyocyte autophagy was accelerated in $m d x$ mice, and that the number of autophagosomes was increased by cardiac stress with isoproterenol. In addition, cardiac fibrosis was increased by autophagy in this context. These data conflicted with prior reports of skeletal muscle autophagy in DMD. Thus, we administered the autophagy 
inhibitor chloroquine to $m d x$ mice to confirm our findings. Chloroquine dramatically decreased cardiomyocyte autophagosome formation and cardiac fibrosis under isoproterenol cardiac stress in $m d x$ mice. These findings suggest that enhanced autophagy is a potential contributor to cardiomyopathy in DMD patients, accelerating cardiomyocyte cell death. These findings were consistent with a recent study demonstrating enhanced cardiac autophagy in DMD, which supports the validity of our findings [22]. Thus, our results, together with prior studies of skeletal muscle, presumably indicate that cellular stress responses are cell type-dependent, and that targeted therapies for DMD should be tailored to the affected organ or cell type.

In clinical settings, cardiac function data are the primary criteria for monitoring cardiomyopathy in DMD patients [23]. Therefore, an echocardiographic approach was used to evaluate cardiac function in $m d x$ mice in the present study. In DMD patients, heart failure follows hypertrophic cardiomyopathy in the early stages, characterized by thinning of the ventricular wall and dilated cardiomyopathy in the late stages [24]. In accordance with clinical parameters were evaluated by echocardiography in $m d x$ mice. Although left ventricular wall thinning and dilatation were not clearly observed in the present study, LVFS and LVEF, indicative of cardiac contractile function, were significantly decreased in $m d x$ mice with isoproterenol cardiac stress. The lack of left ventricular wall thinning and dilatation was likely due to the relatively short duration of our studies, as one month exposure to isoproterenol-induced cardiac stress could be too short for cardiac morphological abnormalities to develop. Nevertheless, cardiac contractile function was impaired by cardiac stress, which was alleviated by chloroquine. These data demonstrated that inhibition of autophagy positively affected cardiac function in this context. These in vivo results suggest the use of chloroquine to improve cardiac function or inhibit cardiac deterioration in DMD patients. In the present study, isoproterenol was used to induce cardiac stress by increasing heart rate. Considering this effect of isoproterenol, combination therapy of chloroquine and beta-blockers could be more effective for treatment of cardiomyopathy in DMD patients.

The clinical application of chloroquine in DMD patients could potentially impair skeletal muscle function, as previous reports have demonstrated that skeletal muscle autophagy is suppressed in DMD $[19,20,21]$. These results are not consistent with the findings of the present study, and suggest a tissue- and cell typespecific role of autophagy in the context of DMD. Further studies are needed to evaluate the potential therapeutic application of chloroquine in DMD, but this modality could potentially reduce skeletal muscle function in DMD patients. Thus, side effects in organs dependent on skeletal muscle function, including the respiratory system, should be carefully evaluated in the clinical application of chloroquine for the treatment of DMD-associated cardiomyopathy. If chloroquine does not adversely affect skeletal muscle function, this modality could be used to treat cardiomyopathy in DMD patients. Alternatively, modalities that specifically inhibit cardiomyocyte autophagy could be developed.

In this study, we focused primarily on autophagy. Cardiomyocyte apoptosis was simultaneously evaluated by using WT and $m d x$ mice. Our findings suggested that cardiomyocyte apoptosis did not significantly differ between these mice. Further studies should be conducted to evaluate the contribution 
of other cell death mechanisms, including necrosis and mitophagy, to cardiomyocyte dysfunction in DMD $[25,26,27]$.

In conclusion, our findings demonstrated that accelerated autophagy was a potential contributing mechanism to cardiomyopathy in the context of DMD. The autophagy inhibitor chloroquine is a potential therapeutic modality for cardiomyopathy of DMD.

\section{Abbreviations}

DMD

Duchenne muscular dystrophy

WT

wild type

iso

isoproterenol

ch

chloroquine

PFA

paraformaldehyde

GFP-mRFP-LC3

green fluorescent protein-LC3

LV

left ventricle

LVDd

left ventricular end-diastolic diameter

LVDs

left ventricular end-systolic diameter

IVSd

end-diastolic interventricular septal thickness

LVPWd

left ventricular end-diastolic posterior wall thickness

LVFS

left ventricular fractional shortening

LVEF

left ventricular ejection fraction

\section{Declarations}

Ethics approval and consent to participate: All animal procedures were conducted in accordance with the guidelines of the Kyoto University Animal Committee and with prior approval from the Institutional Ethical 
Committee and an ARRIVE guideline. Animals were handled in accordance with the Declaration of Helsinki.

Consent for publication: Not applicable.

Availability of data and materials: The datasets used and analyzed during the current study are available from the corresponding author on reasonable request.

Competing interests: The authors declare that they have no competing interests.

Funding: This study was supported by Kiban C Kakenhi, supported by Grant-in-Aid Scientific Research (No. P24791059), Sanofi Pasteur Japan (No. GDC160982), and Novartis Pharma Japan.

Authors' contributions: TH and SB performed most of experiments. SB was a major contributor in writing this manuscript. KA performed western blotting and helped animal experiments. DY and KU analyzed the data. SA analyzed and interpreted the data. TH and JT interpreted the data. All authors read and approved the final manuscript.

Acknowledgements: Not applicable.

\section{Conflicts of Interest}

The authors have no conflicts of interest to declare.

\section{References}

1. Wood S, Shukin RJ, McGillivray BC, Ray PN, Worton RG. A grandpaternally derived de novo deletion within Xp21 initially presenting in carrier females diagnosed as Kugelberg-Welander syndrome. Am J Med Genet. 1988;29(2):419-23.

2. Werner W, Spiegler AW. Inherited deletion of subband Xp21.13 in a male with Duchenne muscular dystrophy. J Med Genet. 1988;25(6):377-82.

3. Emery AE. The muscular dystrophies. Lancet. 2002;359(9307):687-95.

4. Zatz M, Lange K, Spence MA. Frequency of Duchenne muscular dystrophy carriers. Lancet. 1977;1(8014):759.

5. Manzur AYY, Muntoni F. Diagnosis and new treatments in muscular dystrophies. J Neurol Neurosurg Psychiatry. 2009;80:706-14.

6. Patterson V, Morrison O, Hicks E. Mode of death in Duchenne muscular dystrophy. Lancet. 1991;337:801-2.

7. Mukoyama M, Kondo K, Hizawa K, Nishitani H. Life spans of Duchenne muscular dystrophy patients in the hospital care program in Japan. J Neurol Sci. 1987;81:155-8.

8. Emery A, Muntoni F. Management. DUCHENNE MUSCULAR DYSTOROPHY. 3rd Oxford university press. 2003:207-46. 
9. Passamano L, Taglia A, Palladino A, Viggiano E, D'Ambrosio P, Scutifero M, et al. Improvement of survival in Duchenne Muscular Dystrophy: retrospective analysis of 835 patients. Acta Myol. 2012;31:121-5.

10. Eagle M, Baudouin SV, Chandler C, Giddings DR, Bullock R, Bushby K. Survival in Duchenne muscular dystrophy: Improvements in life expectancy since 1967 and the impact of home nocturnal ventilation. Neuromuscul Disord. 2002;12:926-9.

11. Kieny P, Chollet S, Delalande 336 P, Fort M, Le, Magot A, Pereon Y, et al. Evolution of life expectancy of patients with Duchenne muscular dystrophy at AFM Yolaine de Kepper centre between 1981 and 2011. Ann Phys Rehabil Med Elsevier Masson SAS. 2013;56:443-54.

12. Nigro G, Comi LI, Politano L, Bain RJI. The incidence and evolution of cardimyopathy in Duchenne muscular dystrophy. Int J Cardiol. 1990;26:271-7.

13. Politano L, Nigro G. Treatment of dystrophinopathic cardiomyopathy: review of the literature and personal results. Acta Myol myopathies cardiomyopathies Off J Mediterr Soc Myol. 2012;31:24-30.

14. Nigro G, Comi LI, Politano L. Cardiomyopathies associated with muscular dystrophies. In: Engel AG, editor. Myology. 3rd ed. McGraw-Hill; 2004;1239-56.

15. Ogata $H$, Ishikawa $Y$, Ishikawa $Y$, Minami R. Beneficial effects of beta-blockers and angiotensinconverting enzyme inhibitors in Duchennemuscular dystrophy. J Cardiol. 2009;53(1):72 - 8.

16. Viollet L, Thrush PT, Flanigan KM, Mendell JR, Allen HD. Effects of angiotensin-converting enzyme inhibitors and/or beta blockers on the cardiomyopathy in Duchenne muscular dystrophy. Am J Cardiol. 2012;110(1):98-102.

17. Lin B, Li Y, Han L, Kaplan AD, Ao Y, Kalra S, Bett GC, Rasmusson RL, Denning C, Yang L. Modeling and study of the mechanism of dilated cardiomyopathy using induced pluripotent stem cells derived from individuals with Duchenne muscular dystrophy. Dis Model Mech. 2015;8(5):457-66.

18. Young CNJ, Sinadinos A, Lefebvre A, Chan P, Arkle S, Vaudry D, Gorecki DC., A novel mechanism of autophagic cell death in dystrophic muscle regulated by P2RX7 receptor. Autophagy. 2015;11(1):113 $-30$.

19. Whitehead NP, Kim MJ, Bible KL, Adams ME, Froehner SC. A new therapeutic effect of simvastatin revealed by functional improvement in muscular dystrophy. Proc Natl Acad Sci U S A. 2015;112(41):12864-9.

20. Whitehead NP. Enhanced autophagy as a potential mechanism for improved physiological function by simvastatin in muscular dystrophy. Autophagy. 2016;12(4):705-6.

21. Fiacco E, Castagnetti F, Bianconi V, Madaro L, De Bardi M, Nazio F, D'Amico A, Bertini E, Cecconi F, Puri PL, Latella L. Autophagy regulates satellite cell ability to regenerate normal and dystrophic muscles. Cell Death Differ. 2016;23(11):1839-49.

22. Spaulding HR, Ballmann C, Quindry JC, Hudson MB, Selsby JT. Autophagy in the heart is enhanced and independent of disease progression in mus musculus dystrophinopathy models. JRSM Cardiovasc Dis. 2019;8:2048004019879581. doi:10.1177/2048004019879581.

23. Kamdar F, Garry DJ. Dystrophin-Deficient Cardiomyopathy. J Am Coll Cardiol. 2016;67(21):2533-46. 
24. Jefferies JL, Eidem BW, Belmont JW, Craigen WJ, Ware SM, Fernbach SD, Neish SR, Smith EO, Towbin JA. Genetic predictors and remodeling of dilated cardiomyopathy in muscular dystrophy. Circulation. 2005;112(18):2799-804.

25. Kang C, Badr MA, Kyrychenko V, Eskelinen EL, Shirokova N. Deficit in PINK1/PARKIN-mediated mitochondrial autophagy at late stages of dystrophic cardiomyopathy. Cardiovasc Res. 2018;114(1):90-102.

26. Kuno A, Hosoda R, Sebori R, Hayashi T, Sakuragi H, Tanabe M, Horio Y. Resveratrol Ameliorates Mitophagy Disturbance and Improves Cardiac Pathophysiology of Dystrophin-deficient $m d x$ Mice. Sci Rep. 2018;8(1):15555.

27. Capitanio D, Moriggi M, Torretta E, Barbacini P, De Palma S, Viganò A, Lochmüller $H$, Muntoni F, Ferlini A, Mora M, Gelfi C. Comparative proteomic analysis of Duchenne muscular dystrophy and Becker muscular dystorophy muscles: changes contributing to preserve muscle function in Becker muscular dystrophy patients. J Cachexia Sarcopenia Muscle. 2020;11(2):547-63.

\section{Figures}
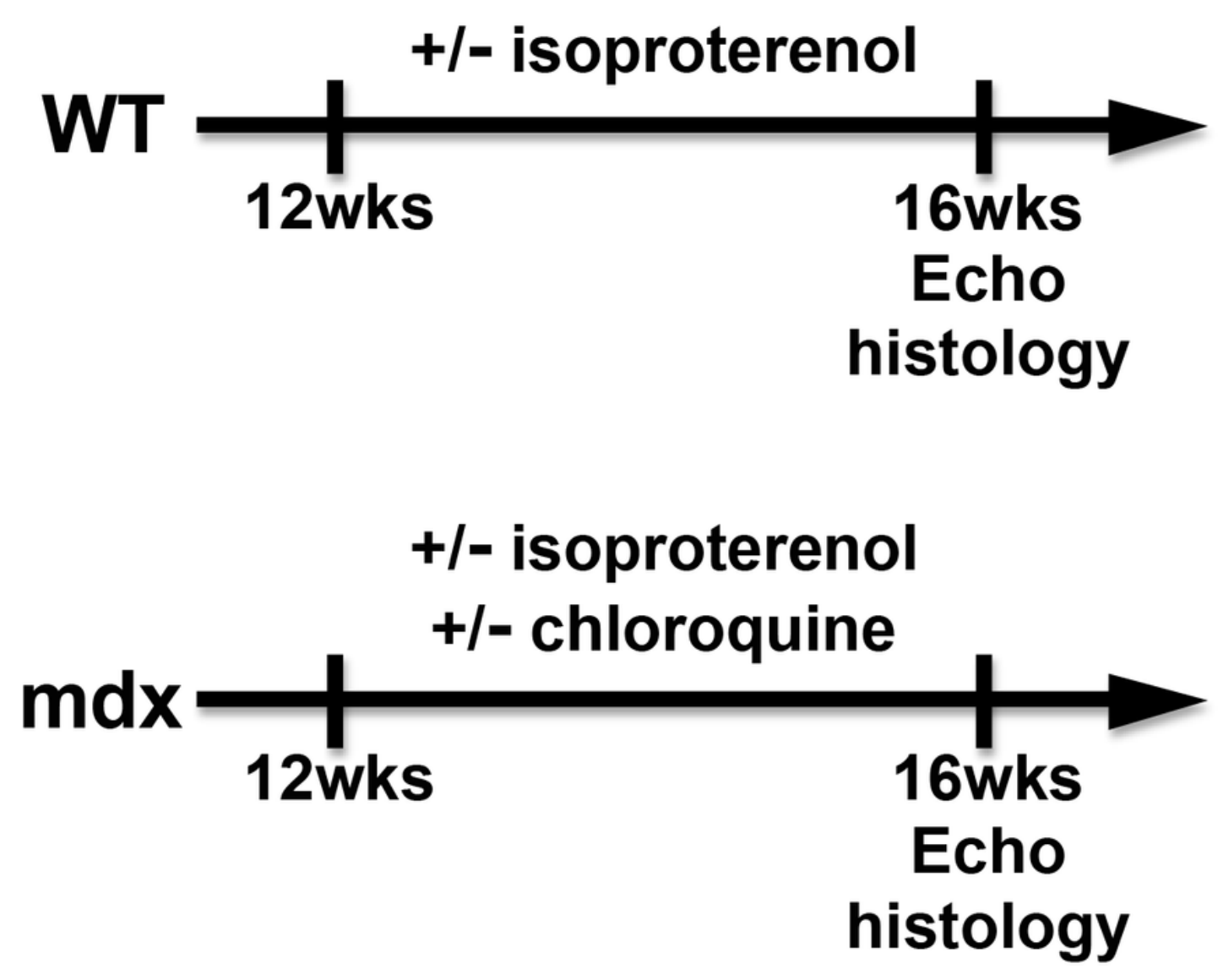


\section{Figure 1}

Animal experiment protocol.
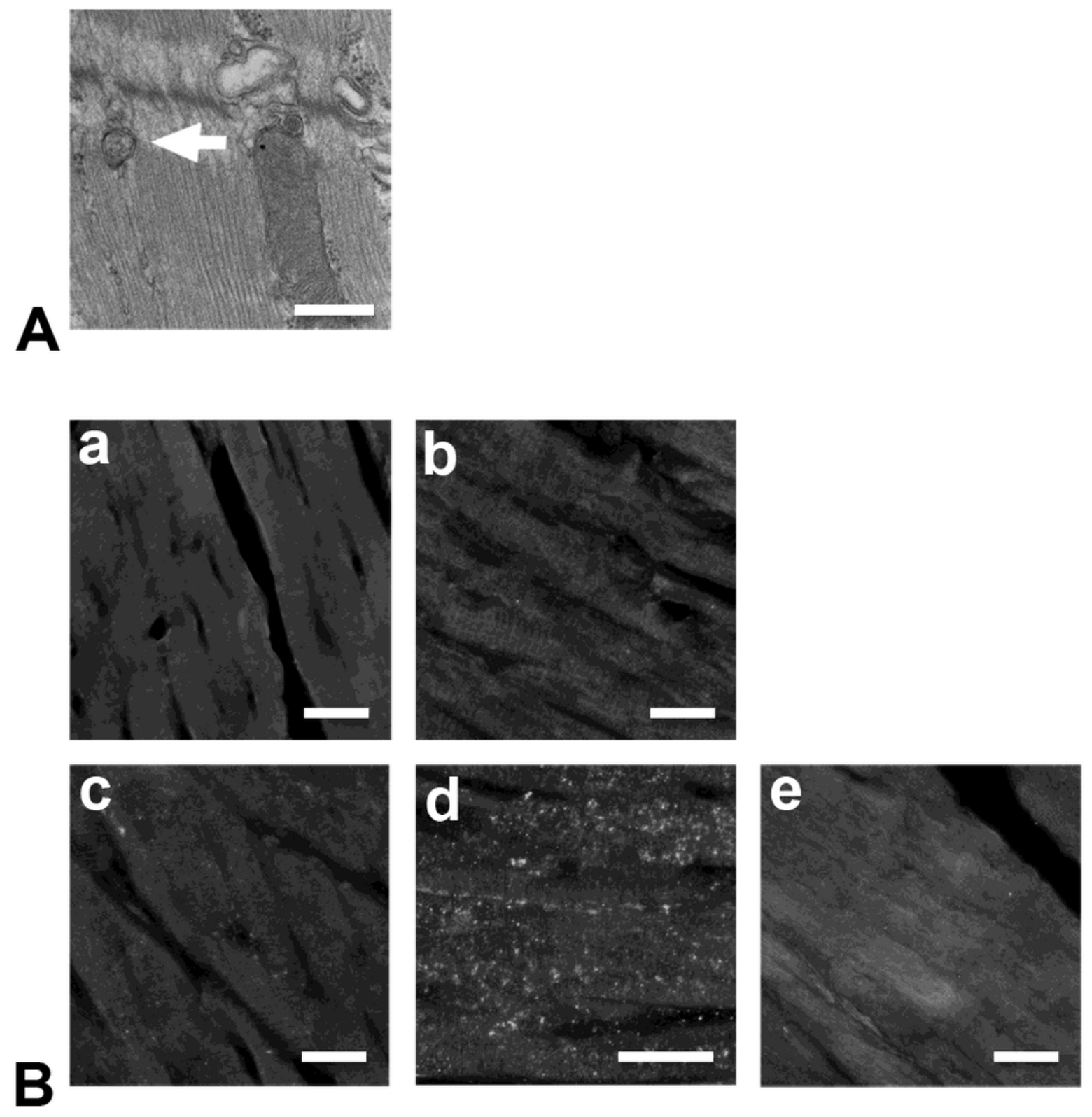

\section{Figure 2}

Autophagosome detection in left ventricle (LV) sections. (A) A representative autophagosome in an LV cardiomyocyte. (Arrow) Scale bar, $100 \mu \mathrm{m}$. (B) GFP-mRFP-LC3 positive autophagosomes in LV sections with and without isoproterenol treatment. a) WT untreated, b) WT isoproterenol-treated, c) mdx untreated, d) $\mathrm{mdx}$ isoproterenol-treated, e) $\mathrm{mdx}$ isoproterenol + chloroquine-treated. Scale bar, $50 \mu \mathrm{m}$. 

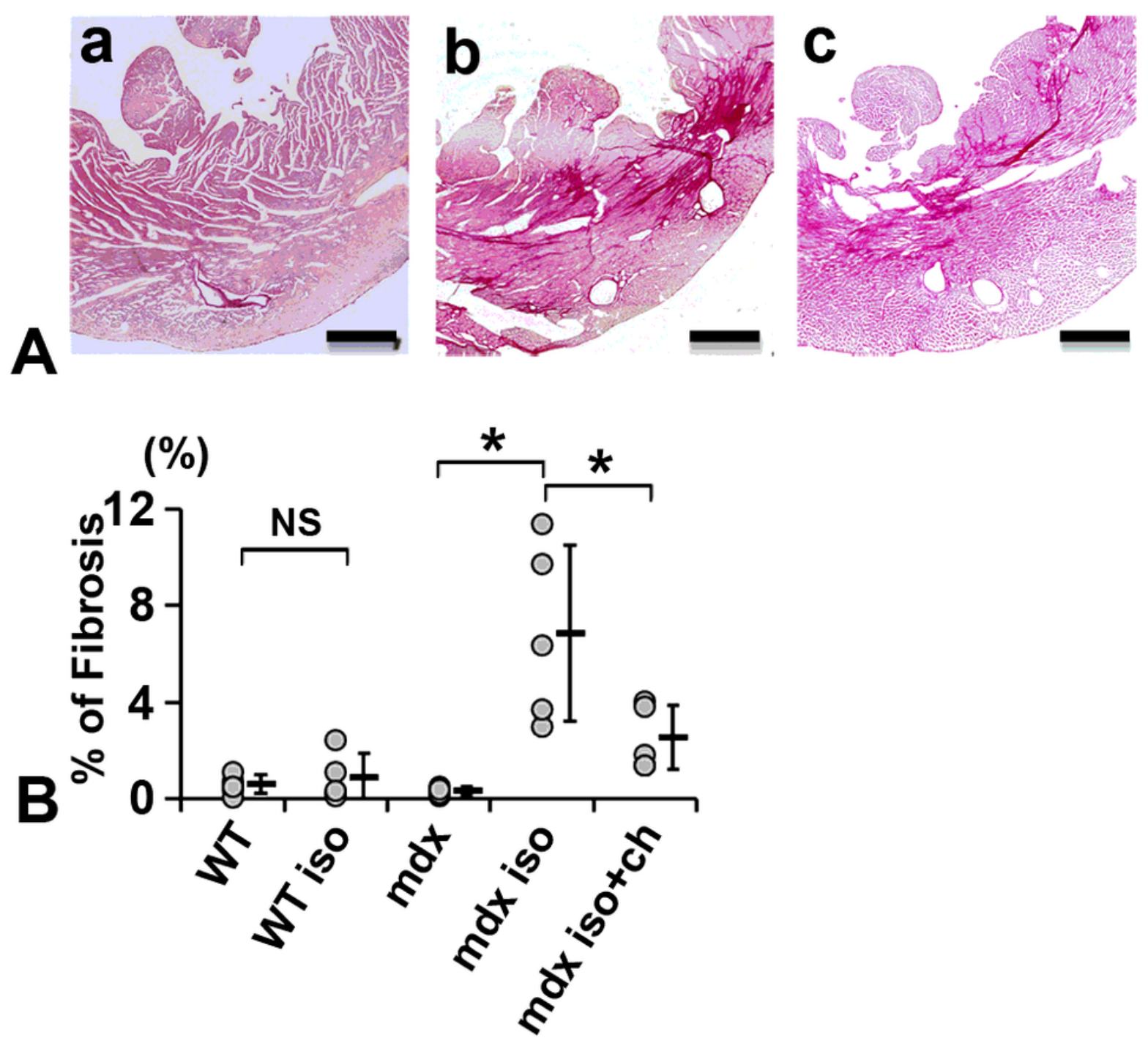

Figure 3

Fibrosis evaluation in LV sections. (A) LV wall fibrosis was detected by Picro-Sirius red staining. Fibrotic tissue is stained as the red area. Representative heart sections of a) an isoproterenol-treated WT mouse, b) an isoproterenol-treated $\mathrm{mdx}$ mouse, and c) an isoproterenol- and chloroquine-treated $\mathrm{mdx}$ mouse heart. Scale bar, $100 \mu \mathrm{m}$. (B) Fibrotic area in LV walls. WT, untreated WT; WT iso, isoproterenol-treated WT; $\mathrm{mdx}$, untreated $\mathrm{mdx}$; $\mathrm{mdx}$ iso, isoproterenol-treated $\mathrm{mdx}$ mice; $\mathrm{mdx}$ iso+ch, isoproterenol- and chloroquinetreated mdx mice. 

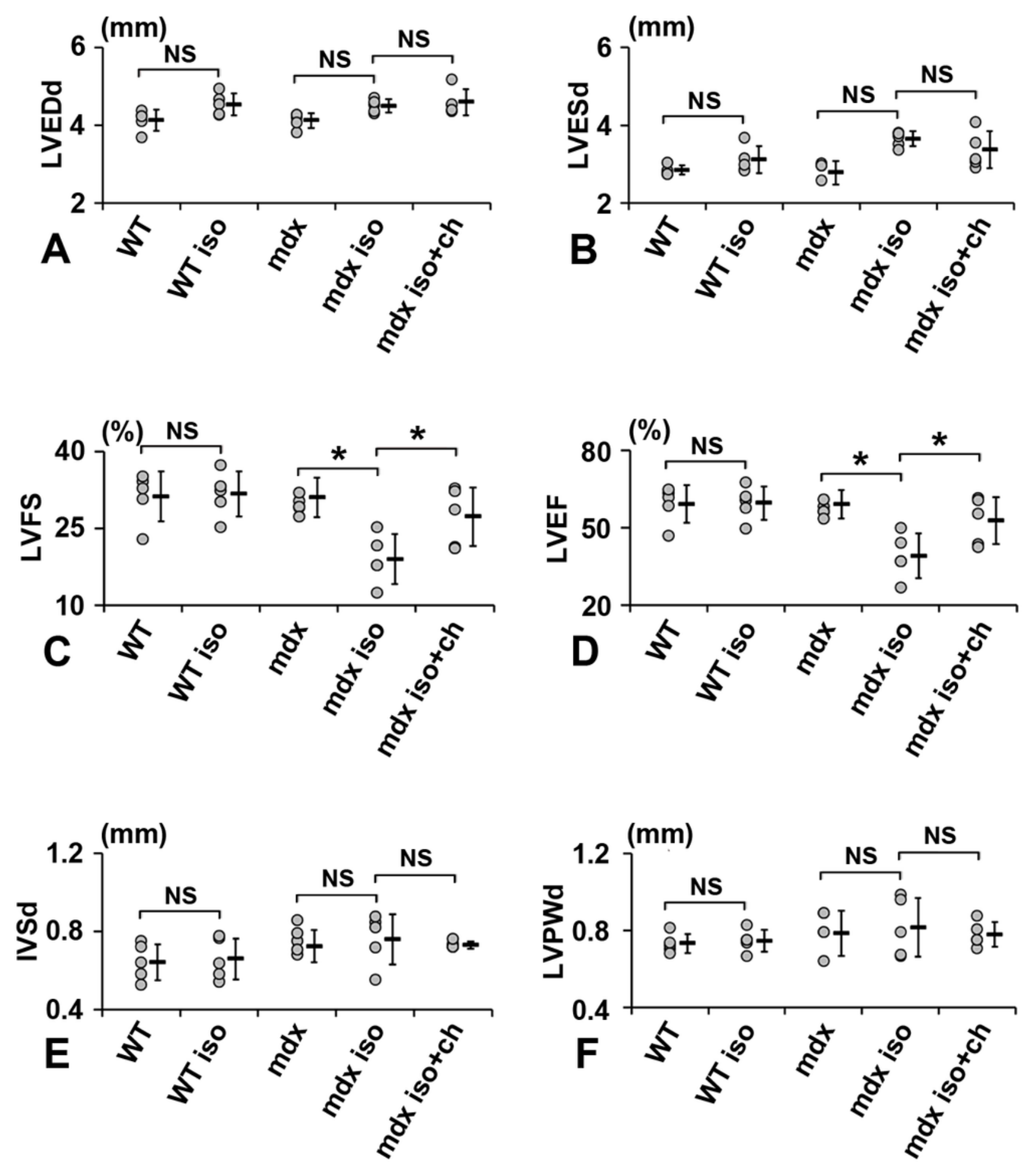

Figure 4

LV functional measurements. (A) LVDd, (B) LVDs, (C) LVFS, (D) LVEF, (E) IVS, and (F) LVPWd were measured by echocardiography. WT, untreated WT; WT iso, isoproterenol-treated WT; mdx, untreated mdx; $\mathrm{mdx}$ iso, isoproterenol-treated $\mathrm{mdx}$ mice; $\mathrm{mdx}$ iso+ch, isoproterenol- and chloroquine-treated $\mathrm{mdx}$ mice. 
This is a list of supplementary files associated with this preprint. Click to download.

- SupFigure120210110SB.tif 